\title{
La vacuna polio oral en lactantes no interfiere con la detección de enterovirus en sangre
}

\author{
Marcela González, Carmen Sandoval, Patricia Valenzuela, Luisa Montecinos, \\ Constanza Martínez, Paula Godoy y Katia Abarca
}

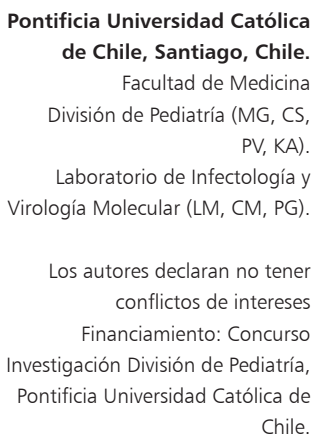

Pontificia Universidad Católica de Chile, Santiago, Chile. Facultad de Medicina División de Pediatría (MG, CS,

$\mathrm{PV}, \mathrm{KA})$.

Laboratorio de Infectología y Virología Molecular (LM, CM, PG).

Los autores declaran no tene conflictos de intereses

Financiamiento: Concurso Investigación División de Pediatría, Pontificia Universidad Católica de

Recibido: 13 de junio de 2013 Aceptado: 9 de octubre de 2013

Correspondencia a: Katia Abarca Villaseca katia@med.puc.cl

\section{Oral polio vaccine in infants does not interfere in detection of enterovirus in blood}

Introduction: There is not known if a viraemia post-oral polio vaccine (OPV) is detectable by modern molecular techniques. Such viraemia could affect the performance of the real time-polymerase chain reaction (PCR) for non polio enterovirus $(\mathrm{EV})$ detection, technique of growing clinical use for the study of febrile infants. Objective: To determine viraemia post-first dose of OPV in healthy infants, by molecular techniques. Patients and Methods: 50 infants less than three months without previous VPO were randomized in 5 groups: a control group with prevaccination blood sample (BS), group 1 BS at day 2, group 2 BS at day 4, group 3, BS at day 6 and group 4, BS at day 8 post-vaccination. Conventional and specific PCR for poliovirus and real time PCR for non polio EV were performed in BS and in OPV samples. Results: No genetic material of poliovirus was detected in any infant, while in 9 of them (18\%) non polio EV was identified. Real time PCR for EV did not amplify poliovirus from OPV samples. Discussion: Results suggest that no post VPO viraemia detectable by molecular methods exists. Considering that real time PCR for EV does not allow to identify polio virus, no false positives of the test are expected as a result of a recent VPO vaccination. We documented presence of non polio EV in blood of healthy asymptomatic infants.

Key words: Poliovirus vaccine viraemia, enterovirus, PCR, real time PCR.

Palabras clave: Viremia poliovirus vaccinal, enterovirus, RPC, RPC tiempo real.

\section{Introducción}

L os enterovirus (EV) son virus pertenecientes a la familia Picornaviridae, con más de 100 serotipos descritos hasta la fecha, 64 de los cuales infectan al hombre. Tradicionalmente fueron clasificados en los grupos Coxsackie A y B, Echo, polio y EV 68-71 en base a sus características moleculares, pero durante la última década han sido reclasificados en grupos: nominados con letras desde la A a la E, grupo parechovirus y grupo poliovirus $^{1,2}$. Los EV no polio han sido reconocidos como agentes etiológicos de una amplia gama de entidades clínicas tales como meningitis, pleurodinia, miocarditis, pericarditis, herpangina, síndrome pie-mano-boca y conjuntivitis; así como de cuadros exantemáticos y enfermedades febriles inespecíficas ${ }^{3-5}$.

Durante las últimas décadas se ha descrito a los EV no polio como causa relevante de cuadros febriles sin foco evidente en lactantes bajo 3 meses de edad. Distintos estudios en lactantes febriles bajo tres meses sin foco clínico, han permitido identificar mediante reacción de polimerasa en cadena (RPC) a EV no polio como agentes etiológicos de $20-26 \%$ de los casos, pudiendo llegar a $50 \%$ en períodos epidémicos ${ }^{6-8}$. En un estudio realizado en nuestra institución durante un año epidémico, se obtuvo RPC positiva para EV no polio en sangre en $47,8 \%$ de 136 lactantes bajo 3 meses con fiebre y sin focalización al examen físico 9 .

En estos cuadros febriles sin focalización en el SNC, la detección de EV no polio en sangre mediante cultivo es de muy bajo rendimiento, mientras que la amplificación génica mediante RPC con transcripción reversa (RPCTR) ha demostrado ser una herramienta de alta utilidad; la tecnología de tiempo real ha permitido tiempos de respuesta reducidos manteniendo elevada la sensibilidad y especificidad de la RPC $8,10,11$.

Por estos antecedentes, la detección de EV no polio en sangre por RPC constituye actualmente el examen de mayor utilidad clínica para el diagnóstico de infecciones enterovirales sin focalización meníngea. Sin embargo, su aplicación clínica podría verse afectada en lactantes pequeños que reciben vacuna polio oral (VPO), en la medida que ésta pudiera asociarse con presencia del virus o su material genético en sangre. Precisamente, la población en la que este examen tendría su mayor indicación corresponde a lactantes en edad de recibir la primera dosis de VPO.

La información relacionada con viremia post-VPO y su identificación por laboratorio es muy escasa y antigua. En los años 50 y 60 se reportó presencia de virus en sangre mediante cultivo en 2 de 11 voluntarios que recibieron virus polio 2 atenuado oral ${ }^{12}$, en 11 de 46 adultos voluntarios que recibieron diferentes dosis de vacuna de virus polio 1 o trivalente ${ }^{13}$ y en 11 de 22 lactantes sanos 
entre 4 y 20 meses de edad seronegativos pocos días tras la vacunación con VPO trivalente ${ }^{14}$. No existen estudios posteriores publicados que determinen ocurrencia de viremia post-vaccinal mediante RPC en lactantes sometidos a vacunación con VPO.

El objetivo del presente estudio fue determinar la presencia de viremia post-VPO, mediante técnicas moleculares, en lactantes sanos que recibieron la primera dosis de esta vacuna.

\section{Pacientes y Métodos}

El estudio fue aprobado por el Comité de Ética de la Escuela de Medicina de la Pontificia Universidad Católica de Chile.

\section{Pacientes}

Previa firma de consentimiento informado por los padres, 50 lactantes sanos bajo tres meses de edad, que aún no recibían la primera dosis de VPO, fueron ingresados al estudio. Se excluyó a todo lactante que presentara alguna patología aguda intercurrente o patología crónica de base. Con la finalidad de reducir la posibilidad de contacto con virus VPO ambiental, se excluyó también a lactantes cuidados fuera de su hogar y a aquellos que tenían hermanos menores de 20 meses o que convivían con niños menores de esa edad. En todos los pacientes se obtuvo una muestra de sangre para determinar presencia de material genético de EV no polio en ella. Los pacientes fueron divididos en forma aleatoria en cinco grupos de 10 pacientes cada uno: cuatro grupos de estudio y un grupo control. La asignación aleatoria se realizó mediante método de sobre cerrado. En el grupo control se obtuvo la muestra de sangre previa a la primera vacunación con VPO. En los cuatro grupos de estudio se obtuvo la muestra de sangre en distintos tiempos post-vacunación: en el grupo 1 al segundo día postvacunación; en el grupo 2 al cuarto día post-vacunación; en el grupo 3 al sexto día post-vacunación y en el grupo 4 al octavo día post-vacunación.

\section{Estudio molecular de viremia por VPO}

Considerando que no se dispone de un método validado para el diagnóstico molecular de virus polio en sangre, se planificó utilizar dos metodologías de amplificación genética: una RPC-TR convencional utilizando partidores de virus polio (amplifica un segmento del extremo 5' NTR conservada del genoma de virus polio) y una RPC de tiempo real dirigida a la detección de EV no polio (amplifica el segmento 5' NTR no codificante del genoma de EV). Ambas técnicas fueron realizadas en el Laboratorio de Infectología y Virología Molecular de la PUC. Los partidores usados se presentan en la Tabla y corresponden a los recomendados en la literatura científica para la detección de virus polio y EV no polio, respectivamente ${ }^{15-17}$.
RPC convencional para virus polio: Se realizó extracción de ARN viral de cada una de las muestras a través de kit de extracción automático MagNA Pure LC RNA Isolation $\mathrm{Kit}{ }^{\circledR}$ (Roche) según protocolo proporcionado por el fabricante, lográndose obtener suficiente material en 48 muestras. Los ARN extraídos fueron amplificados con los partidores indicados en la Tabla 1. La mezcla de reacción fue modificada de la presentada en la bibliografía, con el fin de optimizar las condiciones determinadas en el laboratorio ${ }^{15,16}$. Brevemente, se mezcló $\mathrm{MgCl}_{2}$ (7,5 $\mathrm{mM}), 0,5 \mu \mathrm{M}$ de cada partidor; $40 \mathrm{U}$ de MMLV; $1.5 \mathrm{U}$ de Taq ADN polimerasa. La RPC-TR se realizó como sigue: transcripción reversa a $48^{\circ} \mathrm{C}$ durante $45 \mathrm{~min}$, seguido de denaturación a $94^{\circ} \mathrm{C}$ durante 2 min, continuando con 40 ciclos de $94^{\circ} \mathrm{C}$ por $1 \mathrm{~min}, 57^{\circ} \mathrm{C} 30 \mathrm{seg}, 53^{\circ} \mathrm{C} 30 \mathrm{seg}, 72^{\circ} \mathrm{C}$ $30 \mathrm{seg}$, terminando con extensión de $7 \mathrm{~min}$ a $72^{\circ} \mathrm{C}$. Los fragmentos fueron visualizados tanto en gel de agarosa teñidos con bromuro de etidio como en gel de acrilamida teñidos con plata.

RPC en tiempo real para EV no polio: $\mathrm{El} \mathrm{ARN}$ extraído fue amplificado mediante RPC-TR y RPC anidada en metodología de tiempo real en equipo Light Cycler (Roche) usando un protocolo publicado que incluye 45 ciclos de amplificación ${ }^{17}$. Se logró obtener material adecuado en las 50 muestras. Debido a que esta RPC no permite identificar el tipo de EV amplificado, se realizó análisis de temperatura de melting como una aproximación indirecta para intentar diferenciar distintos tipos de EV. Melting es la temperatura $\left(\mathrm{t}^{\circ}\right)$ a la cual el $50 \%$ de las hebras del fragmento están separadas y es específica para el tamaño y la composición (secuencias) del fragmento; por ejemplo, fragmentos más ricos en $\mathrm{G}$ y $\mathrm{C}$ tendrán una $\mathrm{t}^{\circ}$ mayor que los que son ricos en A y T. Si los fragmentos son de distinto tamaño, y no difieren en composición (secuencias) los más grandes tienen mayor $\mathrm{t}^{\circ}$ de melting que los más pequeños. Esta técnica se usa para ver diferencias entre fragmentos que son similares entre sí $^{18}$.

Adicionalmente, se realizaron ambas metodologías de amplificación en tres muestras de vacuna VPO utilizada en el Programa Nacional de Inmunizaciones de Chile. Como control negativo se utilizó una cepa de EV Coxsackie B5, obtenida del cepario del laboratorio.

Con el fin de prevenir posibles contaminaciones, se incluyeron controles negativos en cada proceso de extracción, transcripción reversa y amplificación; además, las etapas de extracción, preparación de la RPC y amplificación fueron realizadas en habitaciones diferentes.

\section{Resultados}

Mediante la RPC específica para virus polio no se logró la amplificación del virus vacuna en alguna de las muestras analizadas, lo que sí se logró en la muestra de VPO. 
Tabla 1. Partidores utilizados para la identificación de virus polio y EV no polio

$\begin{array}{lll}\text { Ensayo } & \text { Partidor } & \text { Secuencia } \\ \text { RPC-TR convencional para detección de virus polio } & \text { panPV PCR-1 } & \text { TTIAlIGC(AVG)TGICC(A/G)TT(A/G)TT } \\ & \text { panPV PCR-2 } & \text { Read 2001, Kirkpatrick 1996 } \\ \text { RPC-TR y RPC anidada para detección de EV no polio en tiempo real } & \text { EV1 } & \text { CGG CCC CTG AAT CGC GC } \\ & \text { EV2 } & \text { CAC CGG ATG GCC AAT CCA } \\ & \text { EV3 } & \text { CCC CTG AAT GCG GCT AAT } \\ \text { ATT GTC ACC ATA AGC AGC CA }\end{array}$

Mediante la RPC EV no polio se obtuvo amplificación de genoma de EV no polio en 9 de las 50 muestras procesadas $(18 \%)$. Tres $(6 \%)$ de estas muestras positivas correspondieron a pacientes en que la determinación se realizó previa a la administración de VPO. Las otras seis se distribuyeron en todos los grupos de niños vacunados, de la siguiente forma: dos en el segundo día, uno en el cuarto, dos en el sexto y uno en el octavo día post-vacunación. Todos los niños se encontraban asintomáticos al momento de la toma de la muestra. Con el análisis de la curva de temperatura de melting se pudo identificar el tipo de enterovirus en 6 muestras positivas, correspondiendo 4 a echovirus y 2 a coxsackie; en 3 no fue posible determinar el tipo de EV.

Con esta RPC no se logró la amplificación del virus vacuna en tres ocasiones, concluyendo que los partidores utilizados no permiten amplificar el ARN de las cepas vaccinales.

Las dos muestras que no se procesaron mediante la RPC específica de virus polio por no obtener ARN suficiente, resultaron negativas en la RPC EV no polio.

\section{Discusión}

No se obtuvo amplificación de ARN de virus polio vaccinal con la técnica implementada, en muestra alguna de los lactantes vacunados. Una posible explicación para la no amplificación del virus vacuna Sabin por la RPC para $\mathrm{EV}$ en tiempo real radica en que la atenuación del virus vacuna se logra mediante modificaciones en la región 5'UTR no codificante. Como los partidores de la RPC en tiempo real utilizada están dirigidos a un segmento de ARN altamente conservado en todos los tipos de EV, ubicado en esta región 5'UTR no codificante ${ }^{15}$, probablemente, las modificaciones de los virus Sabin en esta región impiden a los partidores utilizados alinearse y amplificar el ARN vaccinal. Por otra parte, la técnica aplicada en este estudio sería específica para detectar EV no polio y por tanto, adecuada para el diagnóstico de infección por EV no polio, aún en lactantes pequeños sometidos a vacunación con VPO.

Un hallazgo secundario y relevante del estudio fue la constatación que el $18 \%$ de los niños, todos lactantes sanos y asintomáticos, presentaron RPC de EV positiva en sangre. Varios estudios han mostrado que las infecciones por EV no polio son frecuentes en lactantes sanos y pueden cursar en forma asintomática ${ }^{7,19,20}$ y que la población más susceptible a infecciones por EV no polio sería los niños bajo 5 años de edad ${ }^{4}$. Aún está por aclararse el rol de los EV no polio como agentes infectantes en lactantes asintomáticos.

Agradecimientos: A las familias que participaron en el estudio. A los residentes de Pediatría, Paula Rojas y M. de los Ángeles Paul, por su colaboración en el reclutamiento de los pacientes. Al personal del Centro Médico San Joaquín por su colaboración en el reclutamiento de los pacientes.

\section{Resumen}

Introducción: No existen estudios que indiquen si la vacuna polio oral (VPO) produce viremia detectable mediante métodos moleculares. Una eventual viremia podría afectar el rendimiento de la RPC tiempo real para detectar enterovirus (EV) no polio, examen de creciente uso clínico en lactantes pequeños con fiebre sin foco. Objetivo: Determinar viremia post VPO en lactantes sanos, por métodos moleculares. Métodos: 50 menores de 3 meses, al momento de recibir su primera VPO se distribuyeron en forma aleatoria en 5 grupos: control, muestra de sangre pre-vacunación; grupo 1 , muestra al $2^{\circ}$ día; grupo 2 , al $4^{\circ}$ día; grupo 3 , al $6^{\circ}$ día y grupo 4 , al $8^{\circ}$ día post-vacunación. Se realizó RPC convencional específica para virus polio y RPC tiempo real para EV no polio en las muestras de sangre y en muestras de VPO. Resultados: No se identificó presencia de material genético de virus polio en lactante alguno, mientras que en $9(18 \%)$ se identificó presencia de EV no polio. La RPC tiempo real para EV no polio no amplificó material genético a partir de las muestras de VPO. Discusión: Los resultados sugieren que no existe viremia post-VPO detectable por métodos moleculares. Considerando que la RPC tiempo real de EV no polio de uso clínico no permite identificar la presencia de virus polio, estos hallazgos indican que no existirán falsos positivos de este examen como resultado de una vacunación VPO reciente. Adicionalmente se documentó presencia de EV no polio en sangre de lactantes asintomáticos. 


\section{Referencias bibliográficas}

1.- Lo CHW, Wu K G, Lin M Ch, Chen Ch J, Min-The Ho D, Tang R B, et al. Application of a molecular method for the classification of human enteroviruses and its correlation with clinical manifestations. J Microbiol Immunol Infect 2010; 43: 354-9.

2.- Tapparel C, Siegrist F, Petty T J, Kaiser L. Picornavirus and enterovirus diversity with associated human diseases. Infection, Genetics and Evolution 2013; 14: 282-93.

3.- Dagan R. Nonpolio enteroviruses and the febrile young infant: epidemiologic, clinical and diagnostic aspects. Pediatr Infect Dis J 1996; 15: 67-71.

4.- Zaoutis T, Klein J. Enteroviral infections. Pediatr Rev 1998; 19 (6): 183-91.

5.- Khetsuriani N, Lamonte-Fowlkes A, Oberst $\mathrm{S}$, Pallansch M A. Enterovirus surveillance-Unites States, 1970-2005. MMWR Surveill Summ 2006; 55: 1-20

6.- Dagan R, Hall C B, Powell K R, Menegus M A. Epidemiology and laboratory diagnosis of infection with viral and bacterial pathogens in infants hospitalized for suspected sepsis. J Pediatr 1989; 115: 351-6.

7.- Byington C, Taggart W, Carroll K, Hilyard D. A polymerase chain reaction-based epidemiologic investigation of the incidence of nonpolio enteroviral infections in febrile and afebrile infants 90 days and younger. Pediatrics 1999; 103 (3): 27-42.

8.- Rittichier K R, Bryan P A, Bassett K E, More. diagnosis and outcomes of enterovirus infections in young infants. Pediatr Infect Dis J 2005; 24 (6): 546-50.

9.- Abarca K, Viviani T, Peña AM, Ibáñez I, Hirsch T, Montecinos L, et al. Infección por enterovirus en lactantes menores de tres meses de edad con fiebre sin foco aparente o con sospecha de infección de sistema nervioso central. Libro de Resúmenes XXIII Congreso Chileno de Infectología 2006.

10.- Kessler H, Santner B, Rabenau H, Berger A, Vince A and Lewinski C. Rapid diagnosis of enterovirus infection by a new one-step reverse transcription-PCR assay. J Clin Microbiol 1997; 35 (4): 976-7.

11.- Tebruegge M, Curtis N. Enterovirus infections in neonates. Semin Fetal Neonatal Med 2009; 14: 222-7.

12.- Sabin A B. Properties and behavior of orally administered attenuated poliovirus vaccine. JAMA 1957; 164: 1216-23.

13.- McKay H W, Fodor A R, Kokko U P. Viremia following the administration of live poliovirus vaccines. Am J Public Health 1963; 53: 274-85.

14.- Horstmann D, Opton E, Klemperer R, Llado B and Vgnec A. Viremia in infants vaccinated with oral poliovirus vaccine (Sabin). Am J Epidemiol 1964; 79 (1): 47-63.

15.- Read S J, Mitchell J L, Fink C G. Light Cycler multiplex PCR for the laboratory diagnosis of common viral infections of the central nervous system. J Clin Microbiol 2001; 39 (9): 3056-9.

16.- Kirkpatrick D R, Nottay B, Yang C F, Yang S J, Mulders M N, Holloway B P, et al. Group-specific identification of polioviruses by PCR using primers containing mixed-base or deoxyinosine residue at positions of codon degeneracy. J Clin Microbiol 1996; 34 (12): 2990-6.

17.- Read S J, Kurtz J B. Laboratory diagnosis of common viral infections of the central nervous system by using a single multiplex PCR screening assay. J Clin Microbiol 1999; 37 (5): 1352-5.

18.- Ririe K M, Rasmussen R P, Wittwer C T. Product differentiation by analysis of DNA melting curves during the polymerase chain reaction. Annal Biochem 1997; 245: 154-60.

19.- Witsø E, Palacios G, Cinek O, Stene L C, Grinde B, Janowitz D, et al. High prevalence of human enterovirus A infections in natural circulation of human enteroviruses. J Clin Microbiol 2006; 44 (11): 4095-100.

20.- Nwachuku N, Gerba C P. Health risks of enteric viral infections in children. Rev Environ Contam Toxicol 2006; 186: 1-56. 